Original Research Paper

\title{
Low-Homology Protein Structural Class Prediction from Secondary Structure Based on Visibility and Horizontal Visibility Network
}

\author{
${ }^{1}$ Zhi-Qin Zhao, ${ }^{2}$ Liang Luo and ${ }^{1}$ Xiao-Yan Liu \\ ${ }^{1}$ College of Science, Xi'an Shiyou University, Xi'an, 18 Second Dianzi Rd, 710065, Shaanxi, China \\ ${ }^{2}$ Department of Mathematics, School of Science, Xi'an University of Posts and Telecommunications, \\ Xi'an 710121, Shaanxi, China
}

Article history

Received: 19-01-2018

Revised: 01-03-2018

Accepted: 10-03-2018

Corresponding Author: Zhi-Qin Zhao

College of Science, Xi'an Shiyou University, Xi'an, 18 Second Dianzi Rd, 710065,

Shaanxi, China

Email: zhiqin_zhao2014@163.com

\begin{abstract}
In this study, based on the predicted secondary structures of proteins, we propose a new approach to predict protein structural classes $(\alpha, \beta, \alpha / \beta, \alpha+\beta)$ for three widely used low-homology data sets. Fist, we obtain two time siries from the chaos game representation of each predicted secondary structure; second, based on two time series, we construct visibility and horizontal visibility network, respectively and generate a set of features using 17 network features; finaly, we predict each protein structure class using support vector machine and Fisher's linear discriminant algorithm, respectively. In order to evaluate our method, the leave one out cross-validating test is employed on three data sets. Results show that our approach has been provided as a effective tool for the prediction of low-homology protein structural classes.
\end{abstract}

Keywords: Protein Structure Class, Secondary Structure, Chaos Game Representation, Visibility and Horizontal Visibility Network, Support Vector Machine, Fisher's Linear Discriminate

\section{Introduction}

The roles of proteins are varied and complex. Levitt and Chothia (1976) first propose the protein structural classes. In their pioneering work, four structural classes of protein, namely all- $\alpha$, all- $\beta, \alpha \beta$ and $\alpha+\beta$ can be obtained. The all- $\alpha$ and all- $\beta$ classes represent structures that consist of mainly $\alpha$-helices and $\beta$-strands, respectively. The $\alpha / \beta$ and $\alpha+\beta$ classes contain both $\alpha$ helices and $\beta$-strands which are mainly interspersed and segregated, respectively (Murzin et al., 1995).

A knowledge of protein structure class is very important in both theoretical and experimental studies in protein science. The information of structure classes has been employed to improve the prediction accuracy of the protein secondary structure (Gromiha and Selvaraj, 1998), to reduce the search space of various possible conformations of the tertiary structure (Carlacci et al., 1991; Bahar et al., 1997). However, for newly-found proteins, the structural class prediction method of automated and accurate are urgently needed. Therefore, the problem of protein structural class prediction is very important towards the protein structure prediction problem. Despite the significance of this problem, when the sequence similarity rate is low, finding the most precise computational method to solve this problem still remains an unsolved problem.

To predict the protein structural class, the current classification methods mainly focus on two aspects: Feature extraction and classification algorithms. The method of feature extraction contains several aspects. Such as physicochemical based information (Dehzangi et al., 2013a; Sharma et al., 2013), structural based information (Yang et al., 2009; 2010; Zhang et al., 2013; Liu and Jia, 2010; Zhang et al., 2011; Ding et al., 2012; Han et al., 2014; Dehzangi et al., 2013b; 2014; Wang et al., 2014). $\mathrm{Yu}$ et al. (2017) use Chous pseudo amino acid composition and wavelet denoising to prediction structural class. From 2014 to now, several papers (Dehzangi et al., 2014; Wang et al., 2014; Jones, 1999; Faraggi et al., 2012) show that the protein secondary structure is significanc to predict protein structural classes. Firstly the features are extracted, secondly all kinds of algorithms can be used to implement the classification prediction, such as Fisher's linear discriminant algorithm (Yang et al., 2009), Support Vector Machine (SVM) (Cai et al., 2003) and so on. 
In this study, based on the predicted protein secondary structure, we attempt to predict the protein structural classes of the three low-homology data sets. Fist, we obtain two time siries from the chaos game representation of each predicted secondary structure, based on two time series, we generate a set of features using 17 network features of visibility or horizontal visibility network. The structure class for each protein is predicted with support vector machine and Fisher's linear discriminant algorithm, respectively. In order to evaluate our approach, the leave one out cross-validating test is employed on three data sets. The result shows that network features are valid features.

\section{Materials and Methods}

\section{Data Sets}

To evaluate our proposed approach, we employe three benchmarks with low sequence identity including 25PDB(the homology-range between 22 and 45\%) (Yang et al., 2009), 1189 (less than 40\% sequence similarities) (Yang et al., 2009) and 640 (with 25\% sequence identity) (Yang et al., 2010), respectively. The data sets in this study and the number of proteins belonging to four structural classes are shown in Table 1 .

\section{Secondary Structure Prediction}

First, we can predict each amino acid in a protein sequence into one of the three secondary structural elements, C (coil), E (strand) and H (helix). For instance, the amino acid sequence of protein $1 \mathrm{~A} 1 \mathrm{~W}$ as follows: MDPFLVLLHSVSSSLSSSELTELKYLCLGRVGKRKL ERVQSGLDLFSMLLEQNDLEPGHTELLRELLASLR RHDLLRRVDDFELEHHHHHH. In this study, if we submit this amino acid sequence to the web server of PSIPRED (http: //globin.bio.warwick.ac.uk/psipred. or http://bioinf.cs.ucl.ac.uk/psipred/) (Jones, 1999), the predicted secondary structure to be returned will be CCHНHНHНHНHНHНCCHНHНHНHНHНHНHНCC СНHНHНСССНHНHНHНHНHССССССССНHНHН ННННННСНННННННННННННННССССC.

\section{Chaos Game Representation of Predicted Secondary}

Fiser et al. (1994) firstly propose the concept of Chaos Game Representation (CGR) of protein structures. Yang and co-workers proposed CGR of predicted protein secondary structure sequence (Yang et al., 2010) to predict protein structure class.

In this study, based on the method of Yang et al. (2010), the CGR of four proteins secondary structure sequence as shown in Fig. 1. The blue points represent the CGR points, the blue edge represents the sides of equilateral triangles. corresponding to the order in the predicted secondary structure, the order of the blue points is saved, but not shown in the figure. We can see that the plotted points tend to be distributed around the sides $\mathrm{HC}$ and $\mathrm{EC}$, respectively, for proteins in the $\alpha$ and $\beta$ classes. However, the points lie around both sides $\mathrm{HC}$ and EC without preference for proteins in the mixture classes.

Each secondary structure sequence generates a distinct $(x, y)$-coordinate sequence of the plotted points. Hence we model a CGR plot as two time series, one composed of the $x$-coordinates, namely $x$-time series and the other of the $y$-coordinates, namely $y$-time series, as shown in Fig. 2.

Recent research showed that the theory of complex network was an effective approach to analyze time series (Lacasa et al., 2008; Luque et al., 2009; Liu et al., 2014). In this study, we hope to reveal some information in the above time series from the perspective of the visibility network (Lacasa et al., 2008) and the horizontal visibility network (Luque et al., 2009).

Visibility Network (VN): Let $\left\{x_{i}\right\}_{i=1,2, \cdots, N}$ be a time series of length $N$. We can obtain a visibility graph from the mapping of a time series of $n$ data into a network of $n$ nodes (where each datum is associated to a specific node and where temporal order is preserved in the node labelling) according to the following visibility criterion: Two arbitrary data $\left(t_{i}, x_{i}\right)$ and $\left(t_{j}, x_{j}\right)$ in the time series have visibility and consequently become two nodes in the associated graph, if any other data $\left(t_{n}, x_{n}\right)$ such that $t_{j}<t_{n}<$ $t_{i}$ fulfills (Lacasa et al., 2008):

$$
x_{n}<x_{i}+\left(x_{j}-x_{i}\right) \frac{t_{n}-t_{i}}{t_{j}-t_{i}}
$$

Some basic properties of the mapping include undirectedness, connectedness (the visibility graph is always connected by definition) and invariance under affine transformations.

Horizontal Visibility Network (HVN): Let $\left\{x_{i}\right\}_{i=1,2, \cdots, N}$ be a time series of length $N$. The algorithm assigns each datum of the series to a node in the network. Two nodes $i$ and $j$ in the network are connected if one can draw a horizontal line in the time series joining $x_{i}$ and $x_{j}$ that does not intersect any intermediate data height. Hence, $i$ and $j$ are two connected nodes if the following geometrical criterion is fulfilled within the time series (Luque et al., 2009):

$$
x_{i}, x_{j}>x_{n}
$$

For all $n$ such that $i<n<j$. As a result, given each time series, its HVN is unweighed, undirected and connected (each node sees at least its nearest neighbors (left-hand side and right-hand side).

Network features: Here, we briefly introduce the considered features, namely network characteristics, that we extract from the visibility network and the horizontal visibility network. The network can be represented by graph. 
Table 1: The number of proteins belonging to four structural classes in the datasets

\begin{tabular}{llllll}
\hline Data set & all- $\alpha$ & all- $\beta$ & $\alpha / \beta$ & $\alpha+\beta$ & Total \\
\hline 25 PDB & 443 & 443 & 346 & 441 & 1673 \\
1189 & 223 & 294 & 334 & 241 & 1092 \\
640 & 138 & 154 & 177 & 171 & 640 \\
\hline
\end{tabular}
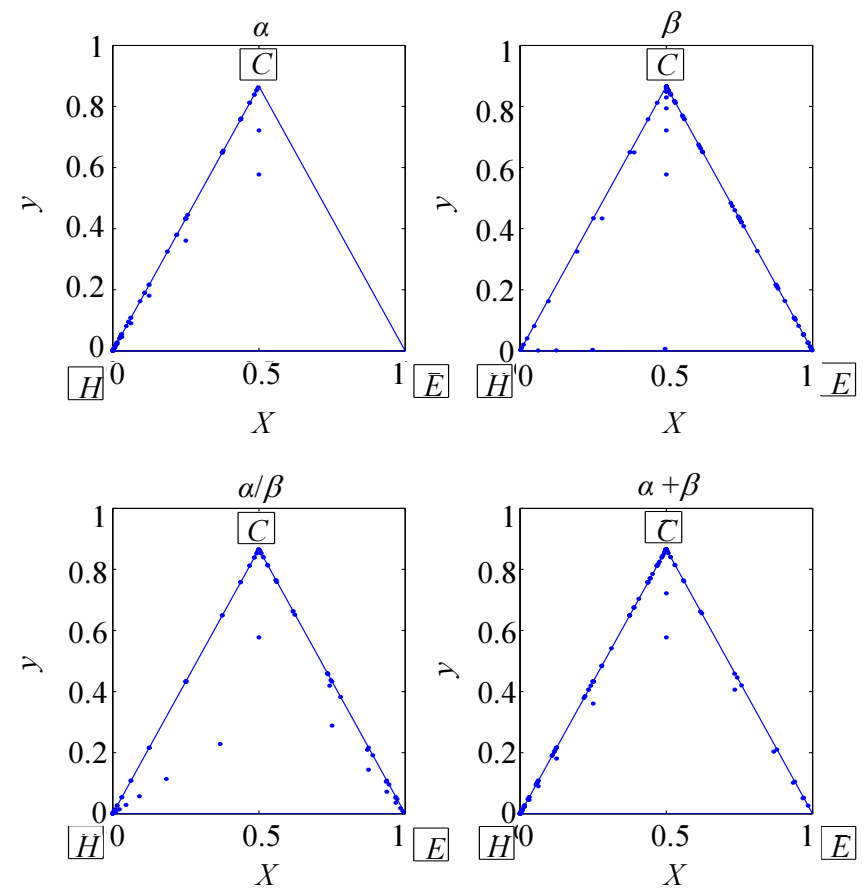

Fig. 1: The CGRs of predicted secondary structure for proteins from four different structural classes. The PDB IDs for four different proteins are $1 \mathrm{~A} 1 \mathrm{~W}(\alpha), 1 \mathrm{~A} 1 \mathrm{X}(\beta), 1 \mathrm{ABA}(\alpha / \beta)$, and 169LA $(\alpha+\beta)$ (Yang et al., 2010)
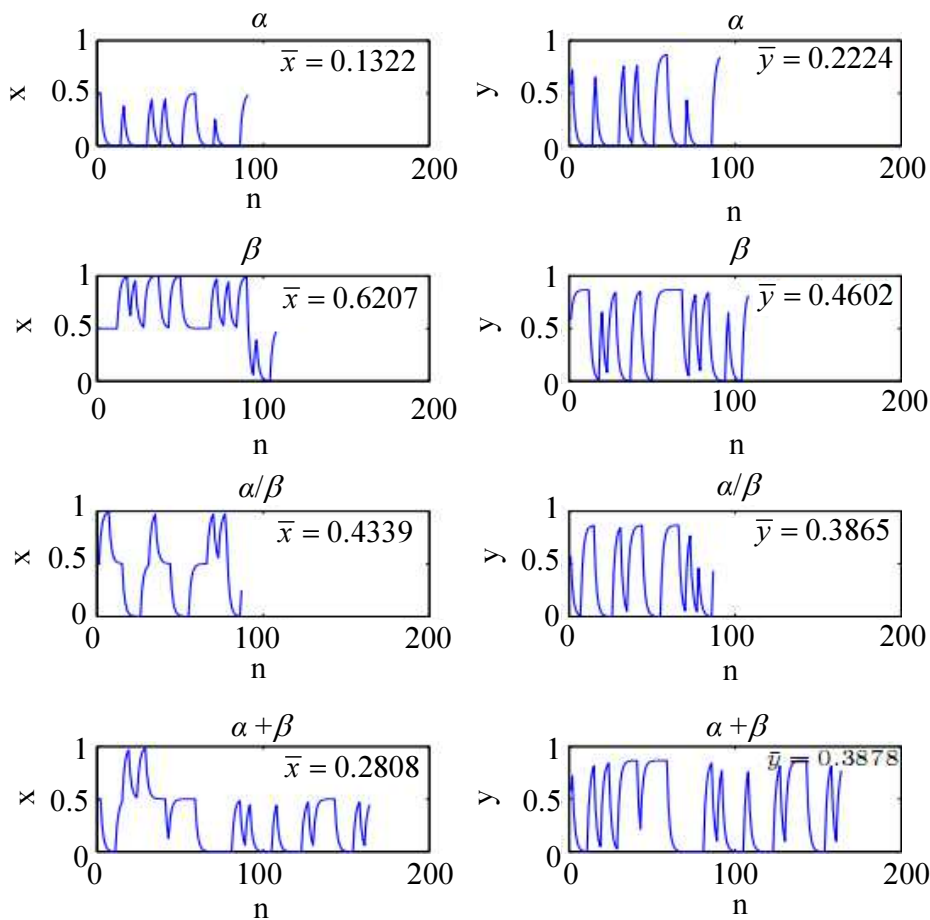

Fig. 2: Eight time series that represent the four CGRs in Fig. 1. Each panel in Fig. 1 gives rise to two time series (x- and ycoordinates, respectively). As a result, we obtain eight time series for four CGRs 
Let $G=(V, E), N=|V|, M=|E|$ be an unweighed and undirected graph, where $N$ and $M$ are the number of nodes and the number of edges, respectively. Let $A$ be the adjacency matrix of the graph $G$.

The number of nodes $(N)$ is an important feature of network.

Average degree $(\bar{K})$ : The degree of any vertex $i$ is given by $K_{i}=\sum_{j=1}^{N} A_{i j}$. The average degree of the network can be written as (Chang et al., 2008) $\bar{K}=\frac{1}{N} \sum_{i} K_{i}$.

Characteristic path length $(L)$ : It is calculated as:

$$
L=\frac{1}{N_{P}} \sum_{j>i} d_{i j}=\frac{1}{N(N-1)} \sum_{i=1} \sum_{j=i+1} d_{i j}
$$

where, $N_{p}$ represents the number of pairs of nodes of the network and $d_{i j}$ is the shortest path (Floyd, 1962) between nodes $i$ and $j$ (Chang et al., 2008). The characteristic path length $L$ is the average of the shortest path lengths.

Diameter (D): The diameter $\mathrm{D}$ is defined as the largest value of all the shortest path lengths in a network. Diameter is a measure of the compactness in a network and is computed by (Emerson and Gothandam, 2012): $D=\operatorname{Max}\left\{d_{i j}\right\}, \forall i-j$ pairs of shortest paths.

Clustering coefficient of the network $(C)$ : The clustering coefficient of any node $i$ is the ratio between the total number of links actually connecting its neighbors and the total number of all possible links between these neighbors. It is given by $C_{i}=\frac{e_{i}}{k_{i}\left(k_{i}-1\right) / 2}$, where $e_{i}$ is the actual number of edges between the neighbors of node $j$. The clustering coefficient of the network is the average of $C_{i}$ overall nodes. It is calculated as (Chang et al., 2008): $C=\frac{1}{N} \sum_{i} C_{i}$.

Pearson correlation coefficient $(r)$ : To understand whether our unweighed undirected networks are of assortive or disassortive type, we calculate the Pearson correlation coefficient $r$ of the degrees at either ends of an edge. For this, we use the expression suggested by Newman (2002):

$$
r=\frac{M^{-1} \sum_{i} j_{i} k_{i}-\left[M^{-1} \sum_{i} 0.5\left(j_{i}+k_{i}\right)\right]^{2}}{M^{-1} \sum_{i} 0.5\left(j_{i}^{2}+k_{i}^{2}\right)-\left[M^{-1} \sum_{i} 0.5\left(j_{i}+k_{i}\right)\right]^{2}}
$$

Here, $j_{i}$ and $k_{i}$ are the degrees of the nodes at the two ends of the $i$-th edge, with $i=1,2, \ldots, M$.
Average closeness centrality $(A C C)$ : Network centrality measures were developed by Freeman (1978; Beauchamp, 1965; Sabidussi, 1966). Basically "closeness centrality" of node $i$ is calculated as:

$$
C C(i)=(N-1) / \sum_{j \in V, j \neq i} d_{i j}
$$

The closeness value is therefore the inverse of the average distance between node $i$ and the other nodes. The average closeness centrality is calculated as:

$$
A C C=\sum_{i} C C(i) / N
$$

Energy (E): The energy (Gutman and Zhou, 2006) of the graph is defined as $E=\sum_{i=1}^{n}\left|\lambda_{i}\right|$, where $\lambda_{i}$ is the ith eigenvalue of the adjacency matrix $A$.

Laplacian Energy $(L E)$ : Let us define the Laplacian matrix as $L=D-A$, where $D$ is a diagonal matrix containing the vertex degrees. The Laplacian energy, $L E$ (Gutman and Zhou, 2006), is defined as:

$$
L E=\sum_{i=1}^{n}\left|\mu_{i}-\frac{2 m}{n}\right|
$$

where, $\mu$ is the $i$-th eigenvalue of the Lappacian.

In this subsection, given a secondary structure sequence, we can convert a protein into two series: $x$ time series and ytime series. Each time series can construct corresponding visibility and horizontal visibility network, respectively. Nine network features can be obtained from a network. The features are the number of nodes $(N)$, average degree $(K)$, characteristic path length $(L)$, network diameter $(D)$, clustering coefficient of network $(C)$, Pearson correlation coefficient $(r)$, average closeness centrality $(A C C)$, Energy $(E)$ and Laplacian Energy $(L E)$. Different time series for the same protein, under the same constructing of network, the number of nodes is the same. Hence we can obtain $1+8 \times 2=17$ features in total for each protein. So, each protein is described as a real-valued vector of 17 features.

\section{Feature Space of Proteins}

As mentioned above, In this study, suppose we use $n$ features to represent a protein sample. Thus, the $i$-th protein sample $P^{i}$ should be a real-valued vector in a $n$-D (dimensional) space, i.e.:

$P^{i}=\left[\begin{array}{llll}p_{1}^{i} & p_{2}^{i} & \cdots & p_{n}^{i}\end{array}\right]^{T}$ 
Here $p_{j}^{i}$ is the $j$-th $(j=1,2, \ldots n)$ feature of the $P^{i}$ and can be derived by following the setps.

Before prediction, each of the $n$ features in Equation (1) should be normalized by:

$$
\begin{aligned}
& p_{j}^{i} \Leftarrow\left(p_{j}^{i}-\mu_{j}\right) / \sigma_{j} \\
& (i=1,2, \cdots, m ; j=1,2, \cdots n)
\end{aligned}
$$

where, $m$ is the number of the total proteins in the data set, $\sigma_{j}=\sqrt{\sum_{i=1}^{m}\left(p_{j}^{i}-\mu_{j}\right)^{2} /(m-1)}$ and $\mu_{j}=\sum_{i=1}^{m} p_{j}^{i} / m$ are the standard and mean deviation of the $j$-th feature over the $m$ protein samples. The normalized values obtained by Equation (2) will have a zero mean value over the $m$ protein samples (Huang et al., 2010).

\section{Support Vector Machine}

Vapnik (1995) introduced a machine learning method of Support Vector Machine (SVM). In our study, we choose Gaussian kernel function. The kernel width parameter $\gamma$ and the regularization parameter $c$ are optimized using a grid search strategy within a limited range, where $\gamma=2^{i}, i=-15,-14,-13, \ldots, 4,5$ and $c=2^{i}, i=$ $-5,-4,-3, \ldots, 14,15$. We find the optimal SVM parameters $c$ and using 10-folding cross validation on the training set for each turn in the leave-one-out cross validation process. The publicly available LIBSVM software (Chang and Lin, 2001) is used to implement the SVM classifier in our paper. The software toolbox can be freely downloaded from http://www.csie.ntu.edu.tw/cjlin/libsvm. Version 3.22 released on December 22, 2016.

\section{Fisher's Discriminant Algorithm}

Fisher's discriminant algorithm (Duda et al., 2001) is used to find a classifier in the parameter space for a training set. A training set $H=\left\{\begin{array}{llll}x_{1} & x_{2} & \ldots & x_{n}\end{array}\right\}$ contains training vectors from two classes. There are $n_{1}$ training vectors from one class forming a subset $H_{1}$ and $n_{2}$ training vectors from another class forming a subset $H_{2}$. Hence, $H=H_{1} \cup H_{2}$ and $n_{1}+n_{2}=n$. Suppose that each $x_{i}$ is a $m$-dimension vector. Then, a parameter vector $\omega$ $=\left(\begin{array}{llll}\omega_{1} & \omega_{2} & \ldots & \omega_{m}\end{array}\right)^{T}$ is estimated such that it allows as many training vectors as possible to be accurately predicted. Specifically:

$$
\begin{aligned}
& m_{j}=\frac{1}{n_{j}} \sum_{x_{i} \in H_{j}} x_{i}, j=1,2 \\
& S_{j}=\sum_{x_{i} \in H_{j}}\left(x_{i}-m_{j}\right)\left(x_{i}-m_{j}\right)^{T}, j=1,2 \\
& S_{\omega}=S_{1}+S_{2}
\end{aligned}
$$

Then the parameter vector $\omega$ is estimated as $S_{\omega}^{-1}\left(m_{1}-m_{2}\right) \quad$ (Duda et al., 2001). By Fisher's discriminant rule, $x$ is assigned to the class of $H_{1}$ if dist $=\left(m_{1}-m_{2}\right)^{T} S_{\omega}^{-1}\left[x-\frac{1}{2}\left(m_{1}+m_{2}\right)\right]>0$ and to the class of $\mathrm{H}_{2}$ otherwise (Duda et al., 2001).

The above algorithm is designed for a two-class problem. In this study, we transform a four-class problem of protein structural classes prediction into six two-class problems, namely, $\alpha$-vs- $\beta, \alpha-\mathrm{vs}-\alpha / \beta, \alpha$ vs- $\alpha+\beta, \beta$-vs- $\alpha / \beta, \beta$-vs- $\alpha+\beta$ and $\alpha / \beta$-vs- $\alpha+\beta$ (Yang et al., 2010).

\section{Performance Evaluation}

The jackknife test (leave-one-out test) (Chou, 1995) is employed in our study.

The individual sensitivity $S_{n}$, the individual specificity $S_{p}$ and the overall accuracy OA over the entire data set, as well as Matthew's correlation coefficient MCC (Xu et al., 2013) are used to evaluate performance.

\section{Results and Discussion}

\section{Prediction Performances of our Method}

The prediction approach is examined with three benchmark data sets in low similarity by leave-one-out test and report the Sensitivity, Specificity and $M C C$ for each structural class, as well as the OA.

By constructing of visibility network, a protein is described as a real-valued vector of 17 features. The results are shown in Table 2. From Table 2, we can see that the overall accuracies for the three data sets are close to or above $80 \%$. Specifically, when SVM is used to implement the classification prediction, the overall accuracies of $82.07,79.03$ and $80 \%$ are achieved for the data sets 25PDB, 1189 and 640, respectively; when Fisher's linear discriminant algorithm is used to implement the classification prediction, the overall accuracies of $80.69,79.40$ and $80 \%$ are achieved for the data sets 25PDB, 1189 and 640, respectively. If comparing the four protein structural classes to each other, the predictions of proteins in the $\alpha$ classes are always the best (with accuracies higher than $90 \%$ for all the data sets.

By constructing of horizontal visibility network, a protein is described as a real-valued vector of 17 features. The results are shown in Table 3. From Table 3, we can see that the overall accuracies for the three data sets are close to or above $80 \%$. Specifically, when SVM is used to implement the classification prediction, the overall accuracies of $82.85 \%, 79.21 \%$ and $81.25 \%$ are achieved for the data sets $25 \mathrm{PDB}$, 
1189 and 640, respectively; when Fisher's linear discriminant algorithm is used to implement the classification prediction, the overall accuracies of 82.19 , 79.30 and $81.41 \%$ are achieved for the data sets $25 \mathrm{PDB}$, 1189 and 640, respectively. If comparing the four protein structural classes to each other, the predictions of proteins in the $\alpha$ classes are always the best (with accuracies higher than $90 \%$ for all the data sets).

From Table 2 and 3, referring to the classes, our method also performs satisfactorily with prediction accuracies of about $80 \%$. However, it seems very challenging to predict the $\alpha / \beta$ class and $\alpha+\beta$ class as their prediction accuracies are relatively low when compared with the other classes.

\section{Comparison with Existing Methods}

In this section, the proposed approach is further compared with other recently reported prediction approachs on the same three data sets. The results are shown in Table 4.

As can be seen from Table 4, our methods obtain the high prediction accuracies for all- $\alpha$, and all- $\beta$ classes among all the tested methods. But our methods obtain the low prediction accuracies for $\alpha / \beta$ and $\alpha+\beta$ classes among all tested methods. But our method shows that network features are useful for prediction of protein structure class.

Table 2: 17 features (VN): The prediction quality of our method on the three data sets with SVM and Fisher algorithms

\begin{tabular}{|c|c|c|c|c|c|c|c|}
\hline \multirow[b]{2}{*}{ Data set } & \multicolumn{4}{|l|}{ SVM } & \multicolumn{3}{|l|}{ Fisher } \\
\hline & Class & Sens & Spec & $\mathrm{MCC}$ & Sens & Spec & $\mathrm{MCC}$ \\
\hline \multirow[t]{5}{*}{ 25PDB } & all- $\alpha$ & 0.9413 & 0.9579 & 0.8906 & 0.9300 & 0.9581 & 0.8828 \\
\hline & all- $\beta$ & 0.8352 & 0.9516 & 0.7988 & 0.8420 & 0.9358 & 0.7793 \\
\hline & $\alpha / \beta$ & 0.7572 & 0.9455 & 0.7179 & 0.7688 & 0.9402 & 0.7171 \\
\hline & $\alpha+\beta$ & 0.7347 & 0.8800 & 0.6040 & 0.6780 & 0.8780 & 0.5545 \\
\hline & $\mathrm{OA}$ & 0.8207 & & & 0.8069 & & \\
\hline \multirow[t]{5}{*}{1189} & all- $\alpha$ & 0.9148 & 0.9565 & 0.8574 & 0.9148 & 0.9595 & 0.8628 \\
\hline & all- $\beta$ & 0.8673 & 0.9530 & 0.8274 & 0.8741 & 0.9472 & 0.8237 \\
\hline & $\alpha / \beta$ & 0.8144 & 0.8704 & 0.6726 & 0.7455 & 0.9169 & 0.6788 \\
\hline & $\alpha+\beta$ & 0.5477 & 0.9002 & 0.4685 & 0.6515 & 0.8690 & 0.5044 \\
\hline & $\mathrm{OA}$ & 0.7903 & & & 0.7940 & & \\
\hline \multirow[t]{5}{*}{640} & all- $\alpha$ & 0.9493 & 0.9744 & 0.9173 & 0.9275 & 0.9821 & 0.9162 \\
\hline & all- $\beta$ & 0.7987 & 0.9534 & 0.7720 & 0.8312 & 0.9389 & 0.7716 \\
\hline & $\alpha / \beta$ & 0.8531 & 0.8848 & 0.7164 & 0.8362 & 0.9055 & 0.7317 \\
\hline & $\alpha+\beta$ & 0.6257 & 0.8862 & 0.5241 & 0.6316 & 0.8745 & 0.5108 \\
\hline & $\mathrm{OA}$ & 0.8000 & & & 0.8000 & & \\
\hline
\end{tabular}

Table 3: About 17 features (HVN): The prediction quality of our method on the three data sets with SVM and Fisher algorithms

\begin{tabular}{|c|c|c|c|c|c|c|c|}
\hline \multirow[b]{2}{*}{ Data set } & \multicolumn{4}{|l|}{ SVM } & \multicolumn{3}{|l|}{ Fisher } \\
\hline & Class & Sens & Spec & $\mathrm{MCC}$ & Sens & Spec & MCC \\
\hline \multirow[t]{5}{*}{$25 \mathrm{PDB}$} & all- $\alpha$ & 0.9549 & 0.9611 & 0.9055 & 0.9436 & 0.9608 & 0.8968 \\
\hline & all- $\beta$ & 0.8014 & 0.9600 & 0.7869 & 0.8397 & 0.9400 & 0.7834 \\
\hline & $\alpha / \beta$ & 0.7890 & 0.9545 & 0.7595 & 0.8064 & 0.9456 & 0.7551 \\
\hline & $\alpha+\beta$ & 0.7596 & 0.8736 & 0.6145 & 0.6939 & 0.8901 & 0.5852 \\
\hline & $\mathrm{OA}$ & 0.8285 & & & 0.8219 & & \\
\hline \multirow[t]{5}{*}{1189} & all- $\alpha$ & 0.9058 & 0.9485 & 0.8360 & 0.9148 & 0.9484 & 0.8424 \\
\hline & all- $\beta$ & 0.8401 & 0.9611 & 0.8191 & 0.8673 & 0.9488 & 0.8207 \\
\hline & $\alpha / \beta$ & 0.7964 & 0.8848 & 0.6762 & 0.7455 & 0.9182 & 0.6806 \\
\hline & $\alpha+\beta$ & 0.6224 & 0.8904 & 0.5151 & 0.6556 & 0.8741 & 0.5160 \\
\hline & $\mathrm{OA}$ & 0.7921 & & & 0.7930 & & \\
\hline \multirow[t]{5}{*}{640} & all- $\alpha$ & 0.9348 & 0.9631 & 0.8855 & 0.9203 & 0.9801 & 0.9070 \\
\hline & all- $\beta$ & 0.8052 & 0.9612 & 0.7904 & 0.8312 & 0.9493 & 0.7887 \\
\hline & $\alpha / \beta$ & 0.8475 & 0.9113 & 0.7485 & 0.8701 & 0.9017 & 0.7531 \\
\hline & $\alpha+\beta$ & 0.6842 & 0.8838 & 0.5690 & 0.6550 & 0.8911 & 0.5556 \\
\hline & $\mathrm{OA}$ & 0.8125 & & & 0.8141 & & \\
\hline
\end{tabular}


Table 4: Performance comparison of different methods on three data sets

\begin{tabular}{|c|c|c|c|c|c|c|}
\hline \multirow[b]{2}{*}{ Data set } & \multirow[b]{2}{*}{ Method } & \multicolumn{5}{|c|}{ Prediction accuracy (\%) } \\
\hline & & all- $\alpha$ & all- $\beta$ & $\alpha / \beta$ & $\alpha+\beta$ & $\mathrm{OA}$ \\
\hline \multirow[t]{13}{*}{$25 \mathrm{PDB}$} & Yang et al. (2009) & 0.5800 & 0.6500 & 0.6990 & 0.6510 & 0.6420 \\
\hline & Yang et al. (2010) & 0.9280 & 0.8330 & 0.8580 & 0.7010 & 0.8290 \\
\hline & Zhang et al. (2013) & 0.9570 & 0.8080 & 0.8240 & 0.7550 & 0.8370 \\
\hline & Liu and Jia (2010) & 0.9260 & 0.8130 & 0.8150 & 0.7600 & 0.8290 \\
\hline & Zhang et al. (2011) & 0.9500 & 0.8560 & 0.8150 & 0.7320 & 0.8390 \\
\hline & Ding et al. (2012) & 0.9503 & 0.8126 & 0.8324 & 0.7755 & 0.8434 \\
\hline & Han et al. (2014) & 0.9460 & 0.8760 & 0.8410 & 0.7820 & 0.8630 \\
\hline & Dehzangi et al. (2014) & 0.9680 & 0.9370 & 0.9010 & 0.8700 & 0.9220 \\
\hline & Wang et al. (2014) & 0.9500 & 0.9140 & 0.7750 & 0.8870 & 0.8880 \\
\hline & This paper VN (SVM) & 0.9413 & 0.8352 & 0.7572 & 0.7347 & 0.8207 \\
\hline & This paper VN (Fisher) & 0.9300 & 0.8420 & 0.7688 & 0.6780 & 0.8069 \\
\hline & This paper HVN (SVM) & 0.9549 & 0.8014 & 0.7890 & 0.7596 & 0.8285 \\
\hline & This paper HVN (Fisher) & 0.9436 & 0.8397 & 0.8064 & 0.6939 & 0.8219 \\
\hline \multirow[t]{13}{*}{1189} & Yang et al. (2009) & 0.6050 & 0.6770 & 0.7100 & 0.6140 & 0.6580 \\
\hline & Yang et al. (2010) & 0.8920 & 0.8670 & 0.8260 & 0.6560 & 0.8130 \\
\hline & Zhang et al. (2013) & 0.9240 & 0.8440 & 0.8440 & 0.7340 & 0.8360 \\
\hline & Liu and Jia (2010) & & & & & \\
\hline & Zhang et al. (2011) & 0.9240 & 0.8740 & 0.8200 & 0.7100 & 0.8320 \\
\hline & Ding et al. (2012) & 0.9372 & 0.8401 & 0.8353 & 0.6639 & 0.8196 \\
\hline & Han et al. (2014) & 0.9100 & 0.8880 & 0.8740 & 0.6930 & 0.8450 \\
\hline & Dehzangi et al. (2014) & 0.9820 & 0.9150 & 0.8380 & 0.7220 & 0.8630 \\
\hline & Wang et al. (2014) & 0.9640 & 0.9290 & 0.8200 & 0.7840 & 0.8710 \\
\hline & This paper VN (SVM) & 0.9148 & 0.8673 & 0.8144 & 0.5477 & 0.7903 \\
\hline & This paper VN (Fisher) & 0.9148 & 0.8741 & 0.7455 & 0.6515 & 0.7940 \\
\hline & This paper HVN (SVM) & 0.9058 & 0.8401 & 0.7964 & 0.6224 & 0.7921 \\
\hline & This paper HVN (Fisher) & 0.9148 & 0.8673 & 0.7455 & 0.6556 & 0.7930 \\
\hline \multirow[t]{13}{*}{640} & Yang et al. (2009) & - & - & - & - & - \\
\hline & Yang et al. (2010) & 0.8910 & 0.8510 & 0.8810 & 0.7140 & 0.8310 \\
\hline & Zhang et al. (2013) & - & - & - & - & - \\
\hline & Liu and Jia (2010) & - & - & - & - & - \\
\hline & Zhang et al. (2011) & - & - & - & - & - \\
\hline & Ding et al. (2012) & 0.9493 & 0.7662 & 0.8927 & 0.7427 & 0.8344 \\
\hline & Han et al. (2014) & - & - & - & - & - \\
\hline & Dehzangi et al. (2014) & - & - & - & - & - \\
\hline & Dehzangi et al. (2014) & 0.9570 & 0.8960 & 0.8930 & 0.9010 & 0.9090 \\
\hline & This paper VN (SVM) & 0.9493 & 0.7987 & 0.8531 & 0.6257 & 0.8000 \\
\hline & This paper VN (Fisher) & 0.9275 & 0.8312 & 0.8362 & 0.6316 & 0.8000 \\
\hline & This paper HVN (SVM) & 0.9348 & 0.8052 & 0.8475 & 0.6842 & 0.8125 \\
\hline & This paper HVN (Fisher) & 0.9203 & 0.8312 & 0.8710 & 0.6550 & 0.8141 \\
\hline
\end{tabular}

\section{Conclusion}

The problem of protein structural class prediction is still a challenge problem. Though some of approachs have shown the state-of-the-art performance, there is always room for improvement. In this study, we used matlab software to write programs. 17 network features are utilized to predict low-homology protein structural class. By comparisons with other existing approachs, we may attribute the high prediction accuracy. Three widely used data sets, 25PDB, 1189 and 640, with low sequence similarity, are adopted to evaluate the performance of our approach. Results by leave-one-out test show that our proposed method provides an effective tool for the accurate prediction of lowhomology protein structural classes.

\section{Acknowledgements}

This work was Supported by National Natural Science Foundation of China (Grant No. 11626187), the Doctoral Scientific Research Foundation of Shaanxi Province (2016BS14), Foundation of Shaanxi Education Committee (No:16JK1603; No:16JK1708) and Shaanxi young science and technology nova (2017KJXX-60).

\section{Author's Contributions}

Zhi-Qin Zhao: Designed and developed the method, performed the numerical experiments, analyzed the data and wrote the paper.

Liang Luo: Performed the numerical experiments and revised the manuscript. 
Xiao-Yan Liu: Revised the manuscript.

\section{Ethics}

The authors declare their responsibility for any ethical issues that may arise after the publication of this manuscript.

\section{Conflict of Interest}

The authors declare that they have no competing interests. The corresponding author affirms that all of the authors have read and approved the manuscript.

\section{References}

Bahar, I., A.R. Atilgan, R.L. Jernigan and B. Erman, 1997. Understanding the recognition of protein structural classes by amino acid composition. Proteins, 29: 172-185. DOI: 10.1002/(SICI)10970134(199710)29:2<172::AID-PROT5>3.0.CO;2-F

Beauchamp, M.A., 1965. An improved index of centrality. Behav. Sci., 10: 161-163. DOI: $10.1002 /$ bs.3830100205

Cai, Y.D., X.J. Liu, X.B. Xu and K.C. Chou, 2003. Support vector machines for prediction of protein domain structural class. J. Theor. Biol., 221: 115-120. DOI: 10.1006/jtbi.2003.3179

Carlacci, L., K.C. Chou and G.M. Maggiora, 1991. A heuristic approach to predicting the tertiary structure of bovine somatotropin. Biochemistry, 30: 4389-4398. DOI: $10.1021 / \mathrm{bi00232a004}$

Chang, C.C. and C.J. Lin, 2001. LIBSVM: A library for support vector machines.

Chang, S., X. Jiao, C. Li, X. Gong and W. Chen et al., 2008. Amino acid network and its scoring application in protein-protein docking. Biophy. Chem., 134: 111-118.

DOI: $10.1016 /$ j.bpc.2007.12.005

Chou, K.C., 1995. A novel approach to predicting protein structural classes in a (20-1)-D amino acid composition space. Proteins, 21: 319-344.

DOI: $10.1002 /$ prot.340210406

Dehzangi, A., K. Paliwal, J. Lyons, A. Sharma and A. Sattar, 2014. Proposing a highly accurate protein structural class predictor using segmentation-based features. BMC Genomics 15: 1-13.

DOI: $10.1186 / 1471-2164-15-S 1-S 2$

Dehzangi, A., K.K. Paliwal, A. Sharma, O. Dehzangi and A. Sattar, 2013a. A combination of feature extraction methods with an ensemble of different classifiers for protein structural class prediction problem. IEEE/ACM Trans. Comput. Biol. Bioinformat., 10: 564-575.

DOI: $10.1109 / \mathrm{TCBB} .2013 .65$
Dehzangi, A., K.K. Paliwal, J. Lyons, A. Sharma and A. Sattar, 2013b. Exploring potential discriminatory information embedded in pssm to enhance protein structural class prediction accuracy. Proceedings of the 8th IAPR International Conference on Pattern Recognition in Bioinformatics, RIB'13, Springer, Berlin, Heidelberg, pp: 208-219. DOI: 10.1007/978-3-642-39159-0 19

Ding, S.Y., S.L. Zhang, Y. Li and T.M. Wang, 2012. A novel protein structural classes prediction method based on predicted secondary structure. Biochim., 94: 1166-1171. DOI: 10.1016/j.biochi.2012.01.022

Duda, R.O., P.E. Hart and D.G. Stork, 2001. Pattern Classification. 2nd Edon., John Wiley and Sons, New York, ISBN-10: 0471056693, pp: 654.

Emerson, A. and K.M. Gothandam, 2012. Network analysis of transmembrane protein structures. Phy. A: Statistical Mechan. Applic., 391: 905-916. DOI: $10.1016 /$ j.physa.2011.08.065

Faraggi, E., T. Zhang, Y. Yang, L. Kurgan and Y. Zhou, 2012. Spine $\mathrm{x}$ : Improving protein secondary structure prediction by multistep learning coupled with prediction of solvent accessible surface area and backbone torsion angles. J. Comput. Chem., 33: 259-267. DOI: 10.1002/jcc. 21968

Fiser, A., G.E. Tusnady and L. Simon, 1994. Chaos game representation of protein structures. J. Mol. Graph., 12: 302-304.

DOI: $10.1016 / 0263-7855(94) 80109-6$

Floyd, R.W., 1962. Algorithm 97: Shortest path. Commun. ACM, 5: 345-345. DOI: 10.1145/367766.368168

Freeman, L.C., 1978. Centrality in social networks conceptual clarification. Social Netw, 1: 215-239. DOI: 10.1016/0378-8733(78)90021-7

Gromiha, M. and S. Selvaraj, 1998. Protein secondary structure prediction in different structural classes. Protein Eng. Design Select., 11: 249-251. DOI: $10.1093 /$ protein/11.4.249

Gutman, I. and B. Zhou, 2006. Laplacian energy of a graph. Linear Algebra Applic., 414: 29-37. DOI: 10.1016/j.laa.2005.09.008

Han, G.S., Z.G. Yu and V. Anh, 2014. Secondary structure element alignment kernel method for prediction of protein structural classes. Curr. Bioinformat., 9: 253-257. DOI: $10.2174 / 1574893609999140523124847$

Huang, T., X.H. Shi, P. Wang, Z.S. He and K.Y. Feng et al., 2010. Analysis and prediction of the metabolic stability of proteins based on their sequential features, subcellular locations and interaction networks. PLoS One, 5: 1-9. DOI: 10.1371/journal.pone.0010972 
Jones, D.T., 1999. Protein secondary structure prediction based on position-specific scoring matrices. J. Mol. Biol., 292: 195-202. DOI: 10.1006/jmbi.1999.3091

Lacasa, L., B. Luque, F. Ballesteros, J. Luque and J. Carlos-Nuno, 2008. From time series to complex networks: The visibility graph. Proc. Natl. Acad. Sci. USA, 105: 4972-4975.

DOI: $10.1073 /$ pnas.0709247105

Levitt, M. and C. Chothia, 1976. Structural patterns in globular proteins. Nature, 261: 552-558. DOI: $10.1038 / 261552 \mathrm{a} 0$

Liu, J.L., Z.G. Yu and V. Ahh, 2014. Topological properties and fractal analysis of recurrence network constructed from fractional Brownian motions. Phys. Rev. E, 89: 1-25.

DOI: 10.1103/PhysRevE.89.032814

Liu, T. and C.Z. Jia, 2010. A high-accuracy protein structural class prediction algorithm using predicted secondary structural information. J. Theor. Biol., 267: 272-275. DOI: 10.1016/j.jtbi.2010.09.007

Luque, B., L. Lacasa, F. Ballesteros and J. Luque, 2009. Horizontal visibility graphs: Exact results for random time series. Phys. Rev. E, 80: 1-17.

Murzin, A.G., S.E. Brenner, T. Hubbard and C. Chothia, 1995. Scop: A structural classification of proteins database for the investigation of sequences and structures. J. Mol. Biol., 247: 536-540. DOI: $10.1016 / \mathrm{S} 0022-2836(05) 80134-2$

Newman, M.E.J., 2002. Assortative mixing in networks. Phys. Rev. Lett., 89: 1-5.

DOI: 10.1103/PhysRevLett.89.208701

Sabidussi, G., 1966. The centrality index of a graph. Psychometrika, 31: 581-603. DOI: $10.1007 / \mathrm{BF} 02289527$

Sharma, A., K.K. Paliwal, A. Dehzangi, J. Lyons and S. Imoto et al., 2013. A strategy to select suitable physicochemical attributes of amino acids for protein fold recognition. BMC Bioinformat., 14: 1-11.

DOI: $10.1186 / 1471-2105-14-233$
Vapnik, V.N., 1995. The Nature of Statistical Learning Theory. 1st Edn., Springer-Verlag New York, ISBN-10: 978-1-4757-2440-0, pp: 188.

Wang, J.R., Y. Li, X. Q. Liu, Q. Dai and Y.H. Yao et al., 2014. High-accuracy prediction of protein structural classes using PseAA structural properties and secondary structural patterns. Biochime, 101: 104-112. DOI: 10.1016/j.biochi.2013.12.021

Xu, Y., J. Ding, L.Y. Wu and K.C. Chou, 2013. iSNOPseAAC: Predict cysteine s-nitrosylation sites in proteins by incorporating position specific amino acid propensity into pseudo amino acid composition. Plos One, 8: 1-7. DOI: 10.1371 /journal.pone.0055844

Yang, J.Y., Z.L. Peng and X. Chen, 2010. Prediction of protein structural classes for low-homology sequences based on predicted secondary structure. BMC Bioinformat., 11: 1-9.

DOI: $10.1186 / 1471-2105-11-S 1-S 9$

Yang, J.Y., Z.L. Peng, Z.G. Yu, R.J. Zhang and V. Anh et al., 2009. Prediction of protein structural classes by recurrence quantification analysis based on chaos game representation. $\mathrm{J}$ Theor. Biol., 257: 618-626.

DOI: $10.1016 /$ j.jtbi.2008.12.027

Yu, B., L.F. Lou and S. Li, 2017. Prediction of protein structural class for low-similarity sequences using Chou's pseudo amino acid composition and wavelet denoising. J. Mol. Graph. Modell., 76: 260-273. DOI: $10.1016 /$ j.jmgm.2017.07.012

Zhang, L.C., X.Q. Zhao and L. Kong, 2013. A protein structural class prediction method based on novel features. Biochimie, 95: 1741-1744. DOI: 10.1016/j.biochi.2013.05.017

Zhang, S., S. Ding and T. Wang, 2011. High-accuracy prediction of protein structural class for lowsimilarity sequences based on predicted secondary structure. Biochimie, 93: 710-714. DOI: 10.1016/j.biochi.2011.01.001 